\title{
Association between polygenic risk score of Alzheimer's disease and plasma phosphorylated tau in individuals from the Alzheimer's Disease Neuroimaging Initiative
}

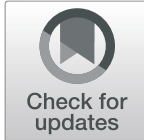

Anna Zettergren ${ }^{1 *}$ D, Jodie Lord² ${ }^{2}$ Nicholas J. Ashton ${ }^{3,4,5,6}$, Andrea L. Benedet ${ }^{7}$, Thomas K. Karikari ${ }^{3}$, Juan Lantero Rodriguez ${ }^{3}$, the Alzheimer's Disease Neuroimaging Initiative* , Anniina Snellman ${ }^{3,8}$, Marc Suárez-Calvet ${ }^{9,10,11,12}$, Petroula Proitsi ${ }^{2}$, Henrik Zetterberg $3,13,14,15$ and Kaj Blennow ${ }^{3,13}$

\begin{abstract}
Background: Recent studies suggest that plasma phosphorylated tau181 (p-tau181) is a highly specific biomarker for Alzheimer's disease (AD)-related tau pathology. It has great potential for the diagnostic and prognostic evaluation of AD, since it identifies AD with the same accuracy as tau PET and CSF p-tau181 and predicts the development of $A D$ dementia in cognitively unimpaired (CU) individuals and in those with mild cognitive impairment (MCl). Plasma p-tau181 may also be used as a biomarker in studies exploring disease pathogenesis, such as genetic or environmental risk factors for AD-type tau pathology. The aim of the present study was to investigate the relation between polygenic risk scores (PRSs) for AD and plasma p-tau181.

Methods: Data from the Alzheimer's Disease Neuroimaging Initiative (ADNI) was used to examine the relation between AD PRSs, constructed based on findings in recent genome-wide association studies, and plasma p-tau181, using linear regression models. Analyses were performed in the total sample $(n=818)$, after stratification on diagnostic status (CU $(n=236), \mathrm{MCl}(n=$ $434)$, AD dementia $(n=148))$, and after stratification on A $\beta$ pathology status (A $\beta$ positives $(n=322)$, A $\beta$ negatives $(n=409)$ ).

(Continued on next page)
\end{abstract}

\footnotetext{
* Correspondence: anna.zettergren@neuro.gu.se

*Data used in the preparation of this article were obtained from the Alzheimer's Disease Neuroimaging Initiative (ADNI) database (http://adni.loni. usc.edu/). As such, the investigators within the ADNI contributed to the design and implementation of ADNI and/or provided data but did not participate in analysis or writing of this report. A complete listing of ADNI investigators can be found at http://adni.loni.usc.edu/wp-content/themes/ freshnews-dev-v2/documents/policy/ADNI_Acknowledgement_List\%205-29-1 8.pdf.

${ }^{1}$ Neuropsychiatric Epidemiology Unit, Department of Psychiatry and Neurochemistry, Institute of Neuroscience and Physiology, the Sahlgrenska Academy, Centre for Ageing and Health (AGECAP) at the University of Gothenburg, Gothenburg, Sweden

Full list of author information is available at the end of the article
}

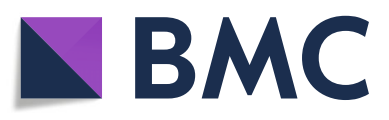

(- The Author(s). 2021 Open Access This article is licensed under a Creative Commons Attribution 4.0 International License, which permits use, sharing, adaptation, distribution and reproduction in any medium or format, as long as you give appropriate credit to the original author(s) and the source, provide a link to the Creative Commons licence, and indicate if changes were made. The images or other third party material in this article are included in the article's Creative Commons licence, unless indicated otherwise in a credit line to the material. If material is not included in the article's Creative Commons licence and your intended use is not permitted by statutory regulation or exceeds the permitted use, you will need to obtain permission directly from the copyright holder. To view a copy of this licence, visit http://creativecommons.org/licenses/by/4.0/. The Creative Commons Public Domain Dedication waiver (http://creativecommons.org/publicdomain/zero/1.0/) applies to the data made available in this article, unless otherwise stated in a credit line to the data. 
(Continued from previous page)

Results: Associations between plasma $p$-tau181 and APOE PRSs $\left(p=3 e^{-18}-7 e^{-15}\right)$ and non-APOE PRSs $\left(p=3 e^{-4}-0.03\right)$ were seen in the total sample. The APOE PRSs were associated with plasma p-tau181 in all diagnostic groups (CU, MCl, and AD dementia), while the non-APOE PRSs were associated only in the MCl group. The APOE PRSs showed similar results in amyloid- $\beta(A \beta)$-positive and negative individuals $\left(p=5 e^{-5}-1 e^{-3}\right.$, while the non-APOE PRSs were associated with plasma $p$-tau181 in $A \beta$ positives only $(p=0.02)$.

Conclusions: Polygenic risk for AD including APOE was found to associate with plasma p-tau181 independent of diagnostic and $A \beta$ pathology status, while polygenic risk for AD beyond APOE was associated with plasma p-tau181 only in $\mathrm{MCl}$ and $\mathrm{A} \beta$-positive individuals. These results extend the knowledge about the relation between genetic risk for AD and p-tau181, and further support the usefulness of plasma p-tau181 as a biomarker of AD.

Keywords: Alzheimer's disease, Polygenic risk score, Plasma phosphorylated tau 181

\section{Background}

Neuropathological features of Alzheimer's disease (AD) are defined by aggregation of amyloid- $\beta$ (A $\beta$ ) protein into plaques and hyperphosphorylation of tau protein with the formation of neurofibrillary tangles [1]. Detection of these processes is crucial for diagnosis of the disease and for recruitment of individuals in clinical trials. The availability of cerebrospinal fluid (CSF) and positron emission tomography (PET) biomarkers has been important for clinical and research work in the field [2]. However, methods for measuring these biomarkers have some drawbacks, since they are invasive, timeconsuming, and expensive; may have side effects; and have limited availability. It is therefore a great need for more easily accessible biomarkers, such as those measured in plasma. Recent studies have indicated that plasma phosphorylated tau 181 (p-tau181) could be a useful biomarker for $\mathrm{AD}$ diagnosis and prognosis. It identifies AD with the same accuracy as tau PET [3-5] and CSF p-tau181 [4] and associates with development of $\mathrm{AD}$ dementia in cognitively unimpaired (CU) individuals and in those with mild cognitive impairment (MCI) [4], as well as in carriers of mutations causing familial $\mathrm{AD}$ [6]. Furthermore, plasma p-tau181 accurately discriminates $\mathrm{AD}$ from non- $\mathrm{AD}$ neurodegenerative disorders with post-mortem confirmation [7]. The relation between plasma p-tau181 and genetic risk for sporadic $\mathrm{AD}$ is, however, uninvestigated.

Genetic risk for $\mathrm{AD}$ is often studied through the use of polygenic risk scores (PRSs), which allow the calculation of genetic risk based on several genetic variants identified through genome wide association studies (GWASs) [8-10]. PRSs for AD have been shown to predict clinical diagnosis [11], pathology-confirmed diagnosis [12], cognitive decline [13], disease progression [14], and imaging biomarkers $[13,15]$. Analyses of the association between AD-PRS and CSF biomarkers have shown somewhat mixed results. The most recent study in the Alzheimer's Disease Neuroimaging Initiative (ADNI) cohort reports that CSF $\mathrm{t}$-tau and $\mathrm{p}$-tau were associated with polygenic scores after correcting for $A P O E \& 4$ status, while the association seen with CSF A $\beta 42$ was driven by $A P O E \varepsilon 4$ homozygotes [16].

There is a need to further explore how genetic risk for $\mathrm{AD}$ influences specific components of the $\mathrm{AD}$ pathophysiology, i.e., brain amyloidosis, tau pathology, and synaptic and neuronal degeneration. Since plasma p-tau181 reflects AD-type tau pathology, it has value as a biomarker to increase understanding on how PRS is linked to tau pathology in AD and thereby increase the knowledge of disease pathogenesis. The aim of the present study was to investigate the relation between AD-PRSs (including and excluding $A P O E$ ) and plasma p-tau181 in ADNI study participants, both in the total sample and after stratifying on diagnostic status and $A \beta$ pathology status.

\section{Methods}

\section{Data}

The data used in this article was obtained from the ADNI database [17]. ADNI was launched in 2003 as a public-private partnership led by Principal Investigator Michael W. Weiner. Briefly, ADNI is a US- and Canadabased longitudinal initiative, following participants through multiple study phases in an attempt to uncover predictive and diagnostic markers of AD [18]. Regional ethical committees of all institutions included in ADNI approved of the study and all subjects have provided informed consent.

\section{Fluid and imaging biomarkers}

Blood samples were collected, processed, stored, and analyzed as described previously [19]. Plasma p-tau181 was measured using the single molecule array (Simoa) technique, using an in-house assay developed in the Clinical Neurochemistry Laboratory, University of Gothenburg, Sweden [3]. This assay uses a combination of two monoclonal antibodies (Tau12 and AT270) and measures Nterminal to mid-domain forms of p-tau181. Further details of the assay can be found in $[3,20]$. Brain $A \beta$ load 
was estimated using $\left[{ }^{18} \mathrm{~F}\right]$ florbetapir (A $\beta$ PET) standardized uptake value ratios (SUVR). SUVR volumes were generated using the full cerebellum as reference region for $\left[{ }^{18} \mathrm{~F}\right]$ florbetapir and amyloid positivity was defined as SUVR > 1.11 [21]. For this study, we used imaging data with the closest acquisition date to the plasma collection. More specifically, amyloid status was given based on $\left[{ }^{18} \mathrm{~F}\right]$ florbetapir scans acquired \pm 180 days (mean (SD): 12.8 (22.1)) from the date of baseline blood collection. Alternatively, a positive amyloid PET status was considered if the PET acquisition was performed more than 180 days before baseline blood collection. Finally, a negative amyloid status was attributed if the PET acquisition was performed more than 180 days after baseline blood collection. Cases that did not fulfill these criteria were excluded.

\section{Genotype data}

Data was available across 1674 individuals and three genotyping platforms: (1) the Human610-Quad platform (ADNI1), (2) the HumanOmniExpress (ADNI2), and (3) Omni 2.5M platform (a combination of ADNI1 and ADNI2 typed at high coverage) [22]. Prior to imputation, data from each of these platforms were QC'ed separately using a combination of PLINK 1.9, PLINK 2.0 [23], and $R$ 3.6.1. Briefly, samples were retained if (1) call rates were above 98\%, (2) identity-by-decent (IBD) scores were lower than 0.1875 (genetic relatedness less than half-way between second and third degree relatives), (3) clinical and genetic sex information corroborated, and (4) samples were of white European descent. To interrogate ancestry beyond that clinically reported, principal components (PCs) were generated using EIGENSOFT 6.1.4 [24] and superimposed onto chart visualizations generated within $\mathrm{R}$ using data from the 1000 genome project [25]. The top 10 PCs were iteratively plotted against each other and visually inspected for each dataset. Upon initial inspection, a sub-cluster of samples were found to cluster independently in PCs explaining the greatest variance within the data. These samples were also found to cluster outside of the European range when superimposed onto 1000 genomes data. Outlier samples were iteratively removed using plot co-ordinates and subsequently re-inspected. Following outlier removal, all data was found to cluster within the European region. Further inspection of sample-only plots indicated a necessity to adjust for the top seven PCs, after which point data was found to fall within a homogenous cluster. Single nucleotide polymorphisms (SNPs), like samples, were retained if call rates were $>98 \%$. Rare SNPs (minor allele frequency $(\mathrm{MAF})<0.01$ ), non-autosomal SNPs, and those deviating from Hardy-Weinberg expectations ( $p$ value threshold of 0.00001 - controls only) were also removed. Any sample overlaps remaining at the end of QC were removed in accordance with the following criteria: for overlaps between Human610-Quad and Omni $2.5 \mathrm{M}$, duplicates in Omni $2.5 \mathrm{M}$ were removed and Human610-Quad retained; for overlaps between HumanOmniExpress and Omni 2.5M, duplicates in HumanOmniExpress were removed and Omni $2.5 \mathrm{M}$ retained, generating a final sample of 1324 individuals.

Imputation was implemented via the Sanger Imputation Service [26], where EAGLE2 was used for prephasing and the Haplotype Reference Consortium was utilized as the reference panel [27]. Post-imputation QC consisted of multi-allelic SNP removals and removal of SNPs with an imputation score $<0.4$ or with dosage certainty $<0.9$. Any retained SNPs were treated as fully observed, and genotypes across the three platforms were merged subsequent to post-imputation QC.

\section{Polygenic risk scores}

Three versions of AD-PRSs were used in this study. To be able to investigate the influence of both "total genetic risk" for $\mathrm{AD}$ and genetic risk for $\mathrm{AD}$ beyond $A P O E$, and to compare our findings with previous studies, all versions were constructed with and without $A P O E$ included. Two of the versions were generated based on summary statistics from the most recent AD GWAS including pure $\mathrm{AD}$ phenotypes [8]. SNPs with $\mathrm{MAF} \geq 5 \%$ were used for selection by linkage disequilibrium (LD)clumping. The European ancestry samples from the 1000 -genomes project were used as reference panel to remove variants in $\mathrm{LD}$, all variants $250 \mathrm{~kb}$ upstream and downstream of the top signal were removed $\left(R^{2}<0.001\right)$. For the non-APOE PRSs, all variants in the $A P O E$ region (chromosome 19, coordinates 44912079 to 45912079) were removed, while the PRRs with $A P O E$ include the variants rs429358 and rs7412. In the present study, we created PRSs including variants based on the $p$ value thresholds $p<5 \mathrm{e}^{-8}$ and $p<1 \mathrm{e}^{-5}$ (referred to as $5 \mathrm{e}^{-8}$ PRS $1 \mathrm{e}^{-5}$ PRS). The third AD-PRS is based on 39 genetic variants (41 variants when including $A P O E$ ) and was validated for the first time in a study by de Rojas et al. [28]. Five of the variants included in the original version of the PRS were missing in the ADNI data, leaving 34 and 36 SNPs respectively. The non- $A P O E$ version of this PRS includes six novel, three confirmed, and 30 previously reported genomewide significant findings. All PRSs were calculated as the sum of the $\beta$-coefficient multiplied with the number of effect alleles of each genetic variant and then standardized. Genetic variants included in the different PRSs are shown in Additional file 1. Median INFO score of the variants used in the $5 \mathrm{e}^{-8}$ PRS and the $1 \mathrm{e}^{-5}$ PRSs was 0.99 (range 0.69-1.00), and for the variants in the 34-SNP PRS it was 0.99 (range 0.591.00). Prior to the PRS calculation 362 individuals who were part of IGAP were removed to avoid overfitting due to nonindependency of the GWAS discovery data and the target 
data, generating a final sample of 962 individuals. We also included the 31-SNP+APOE polygenic hazard score (PHS), originally developed by Desikan et al. [29], that is available in the ADNI database. To facilitate comparison of results based on the PRSs, the PHS was standardized.

\section{Statistical analyses}

Values on plasma p-tau181 were available for 826 of the individuals with PRSs and 822 of those with the PHS. Prior to the statistical analyses, outliers with plasma ptau181 values more than 3 standard deviations (SDs) above or below the average of the whole population with plasma data were excluded ( $n=6$ of those with genetic data), and remaining values were logarithmized to meet normality assumption. Further, two individuals with missing information on diagnostic status in the ADNI database were excluded.

Sample characteristics at the plasma collection baseline (defined as an individual's first visit with plasma data), among individuals with genetic data, were compared using chi-square test (categorical variables) and ANOVA (continuous variables). Associations between AD-PRSs and diagnostic status at plasma collection baseline were investigated using logistic regression models adjusted for sex, age, and seven PCs to correct for population stratification. Associations between ADPRSs and plasma p-tau181 baseline values in the total sample were investigated using linear regression models adjusted for sex, age, diagnostic status $(\mathrm{CU}, \mathrm{MCI}$, or $\mathrm{AD}$ dementia), and seven PCs. Associations between ADPRSs and plasma p-tau181 in the different diagnostic groups were analyzed using linear regression models adjusted for sex, age, and seven PCs. Further, the analyses in the total sample were repeated after stratifying the sample into $A \beta$ positives and negatives. A significance level of $<0.05$ was applied, but the results were also validated in relation to a Bonferroni corrected significance level of $<0.0083$ (based on analyses performed in six different groups (i.e., total sample, $\mathrm{CU}, \mathrm{MCI}, \mathrm{AD}$ dementia, $\mathrm{A} \beta$ positives and negatives). Post hoc power analyses using effect sizes based on the associations seen in this study were performed. The statistical analyses were done in SPSS 26 and SAS 9.4.

\section{Results}

Characteristics of the sample used in this study are shown in Table 1. The diagnostic groups included differed significantly in age, sex, plasma p-tau181 levels, and $A P O E \& 4$ and $\mathrm{A} \beta$ pathology status. Stratification based on $A \beta$ pathology status showed similar results, except that age did not differ between diagnostic groups in $\mathrm{A} \beta$ positives. All versions of APOE PRSs, and the PHS, were associated with both $\mathrm{AD}$ and MCI status. Regarding the non-APOE PRSs, the $1 \mathrm{e}^{-5}$ PRS was associated with $\mathrm{AD}$ and $\mathrm{MCI}$, the 34-SNP PRS was associated with $\mathrm{AD}$, and the $5 \mathrm{e}^{-8}$ PRS was neither associated with $\mathrm{AD}$ nor MCI.

Associations were found between all versions of $A P O E$ PRSs, the PHS, and plasma p-tau181 levels in the total sample (Table 2). Associations, but weaker, were also seen with the non-APOE PRSs, and with the PHS adjusted for $A P O E \& 4$ status (Table 2). When the analyses were repeated after stratifying the sample according to diagnostic status, the APOE PRSs and the PHS were associated with plasma p-tau181 in all groups $(\mathrm{CU}, \mathrm{MCI}$, and AD dementia) (Table 2). The non-APOE PRS, and the PHS adjusted for $A P O E$ \&4 status, showed associations only in the MCI group. All associations, except for those with the non-APOE $1 \mathrm{e}^{-5}$ PRS and the one with the PHS adjusted for $\varepsilon 4$ status in $\mathrm{MCI}$, would remain after Bonferroni correction for multiple testing ( $p$ value limit $<0.0083$ ). Comparing the different versions of PRSs, and the PHS, indicated a relatively similar level of performance, with slightly lower $p$ values for the 34-SNP PRS and the $5 \mathrm{e}^{-8}$ PRS.

Analyses of the total sample stratified into $A \beta$-positive and $A \beta$-negative individuals showed associations between the APOE PRSs, and the PHS, within both groups. The non-APOE PRSs (34-SNP PRS and $1 \mathrm{e}^{-5}$ PRS), and the PHS adjusted for APOE \&4 status, were associated with plasma $\mathrm{p}$-tau181 levels among $\mathrm{A} \beta$ positives only (Table 3). The associations with the $A P O E$ scores, but not those with the non- $A P O E$ scores, would remain after Bonferroni correction for multiple testing ( $p$ value limit $p<0.0083)$. Once again, the different versions of PRSs, and the PHS, performed relatively similar, with slightly lower $p$ values for the 34 -SNP PRS and the $5 \mathrm{e}^{-8}$ PRS. Further, stratification of $A \beta$-positive and $A \beta$-negative individuals based on diagnostic status showed associations between the APOE PRSs, the PHS, and plasma p-tau181 in both $\mathrm{A} \beta$ positives and negatives in all diagnostic groups ( $\mathrm{A} \beta$ positives: $p=5 \mathrm{e}^{-3}-0.02, \mathrm{~A} \beta$ negatives: $p=$ $\left.1 \mathrm{e}^{-3}-0.04\right)$ except for the AD group among $\mathrm{A} \beta$ positives. The non- $A P O E$ scores were borderline associated $(p=$ $0.05-0.07)$ only in $\mathrm{A} \beta$ positives with MCI.

Post hoc power analyses based on effect sizes seen for the associations in this study showed that we had a power between 90 and $>99 \%$ to detect these effects in the total sample, $\mathrm{CU}$ group, and $\mathrm{MCI}$ group, while we had a power of $70 \%$ in the $\mathrm{AD}$ group. In the $\mathrm{A} \beta$ pathology stratified analyses, we had a power between 75 and $>99 \%$.

To investigate if the associations seen between the non-APOE PRSs and plasma ptau-181 were beyond (independent of) $A P O E$, we repeated the analyses of the non- $A P O E$ PRS vs plasma p-tau181 after adjusting for $A P O E \& 4$ status within the regression model. The significant $p$ values within the total sample and the group of 
Table 1 Characteristics of the sample with genetic and plasma p-tau 181 data

\begin{tabular}{|c|c|c|c|c|}
\hline & $\mathrm{CU}(n=236)$ & $\mathrm{MCl}(n=434)$ & $\mathrm{AD}(n=148)$ & \\
\hline & & & & $p^{\mathrm{a}}$ \\
\hline Age in years, mean (SD) & $73.8(6.1)$ & $72.6(7.8)$ & $75.5(7.9)$ & $1 e-4$ \\
\hline Sex (women), $n(\%)$ & $132(55.9)$ & $181(41.7)$ & $56(37.8)$ & $3 e-4$ \\
\hline APOE $\varepsilon 4, n(\%)$ & $69(29.2)$ & $176(40.6)$ & $101(68.2)$ & $2 e-12$ \\
\hline plasma p-tau 181, mean (SD) & $14.9(8.7)$ & $18.3(11.0)$ & $23.6(8.2)$ & $2 e-15$ \\
\hline Years of education, mean (SD) & $16.6(2.7)$ & $16.1(2.8)$ & $16.0(2.7)$ & 0.05 \\
\hline$A \beta$ positivity ${ }^{b}, n(\%)$ & $45(21.0)$ & $176(45.6)$ & $101(77.1)$ & $2 e-23$ \\
\hline APOE PRSs, median (min, max) & & & & $\begin{array}{l}p^{c} \\
p^{d}\end{array}$ \\
\hline 34-SNP PRS & $-0.63(-2.22,2.04)$ & $-0.15(-1.74,2.85)$ & $0.45(-1.94,2.89)$ & - \\
\hline OR $(95 \% \mathrm{Cl})$ & ref & $1.66(1.37-2.02)$ & $2.97(2.24-3.95)$ & $\begin{array}{l}3 e-7 \\
5 e-14\end{array}$ \\
\hline $5 e-8$ PRS & $-0.62(-2.48,1.91)$ & $-0.20(-1.90,2.52)$ & $0.41(-1.83,2.64)$ & - \\
\hline OR $(95 \% \mathrm{Cl})$ & ref & $1.69(1.39-2.05)$ & $2.88(2.18-3.80)$ & $\begin{array}{l}2 e-7 \\
1 e-13\end{array}$ \\
\hline $1 e-5$ PRS & $-0.56(-2.35,2.08)$ & $-0.17(-2.26,2.98)$ & $0.40(-2.08,3.14)$ & - \\
\hline OR $(95 \% \mathrm{Cl})$ & ref & $1.73(1.42-2.11)$ & $3.20(2.37-4.31)$ & $\begin{array}{l}4 e-8 \\
2 e-14\end{array}$ \\
\hline PHS & $-0.66(-2.39,2.03)$ & $-0.23(-1.89,2.45)$ & $0.48(-1.96,2.78)$ & - \\
\hline OR $(95 \% \mathrm{Cl})$ & ref & $1.76(1.44-2.15)$ & $3.31(2.46-4.45)$ & $\begin{array}{l}3 e-8 \\
2 e-15\end{array}$ \\
\hline non-APOE PRSs, median (min, max) & & & & $\begin{array}{l}p^{c} \\
p^{d}\end{array}$ \\
\hline 34-SNP PRS & $-0.14(-2.49,2.88)$ & $-0.02(-2.32,2.75)$ & $-0.03(-2.69,3.62)$ & - \\
\hline OR $(95 \% \mathrm{Cl})$ & ref & $1.11(0.94-1.30)$ & $1.26(1.02-1.56)$ & $\begin{array}{l}0.2 \\
\mathbf{0 . 0 4}\end{array}$ \\
\hline $5 e-8$ PRS & $-0.12(-2.36,2.92)$ & $-0.02(-3.33,3.06)$ & $-0.01(-2.47,3.12)$ & - \\
\hline OR $(95 \% \mathrm{Cl})$ & ref & $1.13(0.96-1.33)$ & $1.21(0.97-1.51)$ & $\begin{array}{l}0.2 \\
0.1\end{array}$ \\
\hline $1 e-5$ PRS & $-0.21(-2.58,2.61)$ & $-0.02(-3.43,2.90)$ & $0.08(-2.53,2.82)$ & - \\
\hline OR $(95 \% \mathrm{Cl})$ & ref & $1.21(1.02-1.42)$ & $1.34(1.07-1.68)$ & $\begin{array}{l}0.03 \\
0.01\end{array}$ \\
\hline$A \beta \operatorname{pos}(n=322)$ & & & & $p^{a}$ \\
\hline Age in years, mean (SD) & $72.2(5.3)$ & $73.9(6.8)$ & $74.0(7.9)$ & 0.5 \\
\hline Sex (women), $n(\%)$ & $32(71.1)$ & $70(39.8)$ & $45(44.6)$ & $1 e-3$ \\
\hline APOE $\varepsilon 4, n(\%)$ & $25(55.6)$ & $117(66.5)$ & $81(80.2)$ & $6 e-3$ \\
\hline Plasma p-tau 181, mean (SD) & $18.5(7.8)$ & $23.0(10.8)$ & $25.0(8.4)$ & $1 e-3$ \\
\hline Years of education, mean (SD) & $16.2(2.5)$ & $15.9(2.8)$ & $15.9(2.7)$ & 0.8 \\
\hline$A \beta$ neg $(n=409)$ & & & & $p^{a}$ \\
\hline Age in years, mean (SD) & $73.3(6.3)$ & $71.1(8.0)$ & $78.1(6.2)$ & $9 e-7$ \\
\hline Sex (women), n (\%) & $85(50.3)$ & $91(43.3)$ & $6(20.0)$ & $8 e-3$ \\
\hline$A P O E \varepsilon 4, n(\%)$ & $37(21.9)$ & $74(35.2)$ & $9(30.0)$ & 0.02 \\
\hline Plasma p-tau 181, mean (SD) & $14.3(8.9)$ & $14.5(9.6)$ & $19.2(7.1)$ & 0.02 \\
\hline Years of education, mean (SD) & $16.7(2.7)$ & $16.4(2.7)$ & $16.2(2.6)$ & 0.4 \\
\hline
\end{tabular}

Significant $p$ values are shown in bold

a Based on ANOVA for continuous variables and chi-square test for categorical variables

${ }^{\mathrm{b}} \mathrm{A} \beta$ pathology status was missing for 87 individuals

${ }^{c}$ Based on logistic regression including cognitively unimpaired (ref) and $\mathrm{MCl}$

${ }^{d}$ Based on logistic regression including cognitively unimpaired (ref) and $A D$ 
Table 2 AD-PRSs versus log plasma p-tau181 in the total sample and by diagnostic status

\begin{tabular}{|c|c|c|c|c|c|c|c|c|}
\hline \multirow[b]{3}{*}{ PRS version } & \multicolumn{4}{|c|}{ APOE PRS } & \multicolumn{4}{|c|}{ Non-APOE PRS } \\
\hline & \multicolumn{8}{|c|}{ Total sample $(n=818)$} \\
\hline & $\beta$ & SE & $95 \% \mathrm{Cl}$ & $p$ & $\beta$ & SE & $95 \% \mathrm{Cl}$ & $p$ \\
\hline 34-SNP PRS & 0.19 & 0.02 & $0.14-0.23$ & $2 e-17$ & 0.06 & 0.02 & $0.02-0.10$ & $2 e-3$ \\
\hline $5 e-8$ PRS & 0.19 & 0.02 & $0.15-0.23$ & $3 e-18$ & 0.08 & 0.02 & $0.03-0.12$ & $3 e-4$ \\
\hline $1 \mathrm{e}-5$ PRS & 0.18 & 0.02 & $0.13-0.22$ & $8 e-16$ & 0.05 & 0.02 & $0.004-0.09$ & 0.03 \\
\hline \multirow[t]{3}{*}{$\mathrm{PHS}^{*}$} & 0.18 & 0.02 & $0.13-0.22$ & $7 e-15$ & 0.11 & 0.04 & $0.03-0.18$ & $8 e-3$ \\
\hline & \multicolumn{8}{|c|}{ Cogn unimpaired $(n=236)$} \\
\hline & $\beta$ & SE & $95 \% \mathrm{Cl}$ & $p$ & $\beta$ & SE & $95 \% \mathrm{Cl}$ & $p$ \\
\hline 34-SNP PRS & 0.16 & 0.05 & $0.05-0.27$ & $3 e-3$ & 0.02 & 0.04 & -0.07 to 0.10 & 0.7 \\
\hline $5 e-8$ PRS & 0.20 & 0.05 & $0.09-0.31$ & $3 e-4$ & 0.08 & 0.04 & -0.003 to 0.17 & 0.06 \\
\hline $1 e-5$ PRS & 0.18 & 0.06 & $0.07-0.28$ & $2 e-3$ & 0.04 & 0.05 & -0.05 to 0.13 & 0.4 \\
\hline \multirow[t]{3}{*}{$\mathrm{PHS}^{*}$} & 0.19 & 0.06 & $0.08-0.30$ & $1 e-3$ & 0.13 & 0.10 & -0.08 to 0.33 & 0.2 \\
\hline & \multicolumn{8}{|c|}{$\mathrm{MCl}(n=434)$} \\
\hline & $\beta$ & SE & $95 \% \mathrm{Cl}$ & $p$ & $\beta$ & SE & $95 \% \mathrm{Cl}$ & $p$ \\
\hline 34-SNP PRS & 0.21 & 0.03 & $0.15-0.27$ & $8 e-13$ & 0.09 & 0.03 & $0.04-0.15$ & $1 e-3$ \\
\hline $5 e-8$ PRS & 0.21 & 0.03 & $0.15-0.26$ & $2 e-12$ & 0.08 & 0.03 & $0.03-0.14$ & $1 e-4$ \\
\hline $1 \mathrm{e}-5$ PRS & 0.19 & 0.03 & $0.14-0.25$ & $4 e-11$ & 0.06 & 0.03 & $0.004-0.12$ & 0.04 \\
\hline \multirow[t]{3}{*}{$\mathrm{PHS}^{*}$} & 0.18 & 0.03 & $0.13-0.24$ & $2 e-9$ & 0.11 & 0.06 & $0.004-0.22$ & 0.04 \\
\hline & \multicolumn{8}{|c|}{$\mathrm{AD}(n=148)$} \\
\hline & $\beta$ & SE & $95 \% \mathrm{Cl}$ & $p$ & $\beta$ & SE & $95 \% \mathrm{Cl}$ & $p$ \\
\hline 34-SNP PRS & 0.13 & 0.03 & $0.07-0.19$ & $6 e-5$ & 0.03 & 0.03 & -0.03 to 0.09 & 0.4 \\
\hline $5 e-8$ PRS & 0.12 & 0.03 & $0.06-0.18$ & $7 e-5$ & 0.03 & 0.03 & -0.04 to 0.09 & 0.4 \\
\hline $1 e-5$ PRS & 0.13 & 0.03 & $0.07-0.19$ & $7 e-5$ & 0.03 & 0.03 & -0.04 to 0.09 & 0.4 \\
\hline $\mathrm{PHS}^{*}$ & 0.12 & 0.03 & $0.06-0.18$ & $1 e-4$ & 0.06 & 0.05 & -0.04 to 0.15 & 0.2 \\
\hline
\end{tabular}

Results within the total sample are adjusted for sex, age, principal components, and diagnostic status. Results within the diagnostic groups are adjusted for sex, age, and principal components. Significant $p$ values are shown in bold

*Values presented in the "non-APOE PRS column" are adjusted for $\varepsilon 4$-carriership instead of excluding APOE

Table 3 AD-PRSs versus log plasma p-tau181 in A $\beta$-positive and A $\beta$-negative individuals

\begin{tabular}{|c|c|c|c|c|c|c|c|c|}
\hline \multirow[b]{3}{*}{ PRS version } & \multicolumn{4}{|c|}{ APOE PRS } & \multicolumn{4}{|c|}{ Non-APOE PRS } \\
\hline & \multicolumn{8}{|c|}{$\mathrm{A} \beta \operatorname{pos}(n=322)$} \\
\hline & $\beta$ & SE & $95 \% \mathrm{Cl}$ & $p$ & $\beta$ & SE & $95 \% \mathrm{Cl}$ & $p$ \\
\hline 34-SNP PRS & 0.10 & 0.03 & $0.05-0.15$ & $7 e-5$ & 0.06 & 0.02 & $0.01-0.10$ & 0.02 \\
\hline $5 e-8$ PRS & 0.10 & 0.03 & $0.05-0.15$ & $9 e-5$ & 0.05 & 0.02 & $0.01-0.10$ & 0.02 \\
\hline $1 e-5$ PRS & 0.10 & 0.03 & $0.05-0.15$ & $2 e-4$ & 0.05 & 0.02 & -0.001 to 0.09 & 0.06 \\
\hline \multirow[t]{3}{*}{$\mathrm{PHS}^{*}$} & 0.10 & 0.03 & $0.05-0.22$ & $2 e-4$ & 0.09 & 0.04 & $0.01-0.17$ & 0.02 \\
\hline & \multicolumn{8}{|c|}{ A $\beta$ neg $(n=409)$} \\
\hline & $\beta$ & SE & $95 \% \mathrm{Cl}$ & $p$ & $\beta$ & SE & $95 \% \mathrm{Cl}$ & $p$ \\
\hline 34-SNP PRS & 0.15 & 0.04 & $0.07-0.23$ & $2 e-4$ & 0.01 & 0.03 & -0.05 to 0.08 & 0.7 \\
\hline $5 e-8$ PRS & 0.17 & 0.04 & $0.09-0.25$ & $5 e-5$ & 0.04 & 0.04 & -0.04 to 0.10 & 0.3 \\
\hline $1 e-5$ PRS & 0.14 & 0.04 & $0.06-0.22$ & $1 e-3$ & -0.003 & 0.03 & -0.07 to 0.06 & 0.9 \\
\hline $\mathrm{PHS}^{*}$ & 0.14 & 0.04 & $0.06-0.15$ & $1 e-3$ & 0.08 & 0.08 & -0.07 to 0.24 & 0.3 \\
\hline
\end{tabular}

Results are adjusted for sex, age, principal components, and diagnostic status. Significant $p$ values are shown in bold *Values presented in the "non-APOE PRS column" are adjusted for $\varepsilon 4$-carriership instead of excluding APOE 
$\mathrm{A} \beta$ positives remained at exactly the same level, while significant $p$ values within the MCI-group increased slightly (non- $A P O E$ 34-SNP PRS: $p=3 \mathrm{e}^{-3}$, non- $A P O E$ $5 \mathrm{e}^{-8}$ PRS: $\left.p=8 \mathrm{e}^{-3}\right)$. To study this in even more detail, we performed stratified analyses, based on $A P O E$ \&4 carrier status, within the total sample. We found that the non- $A P O E$ 34-SNP PRS and the non- $A P O E 5 \mathrm{e}^{-8}$ PRS were significantly associated with plasma p-tau181 in both $\varepsilon 4$-carriers $(p=0.02-0.04)$ and non-carriers $(p=$ $\left.7 \mathrm{e}^{-3}-0.03\right)$.

\section{Discussion}

In this study, we investigated the relation between $\mathrm{AD}$ PRSs and plasma p-tau181. Associations with both $A P O E$ PRSs and non-APOE PRSs were seen in the total sample including both symptomatic (i.e., $\mathrm{MCI}$ and $\mathrm{AD}$ dementia) and cognitively unimpaired individuals. The associations with the non-APOE PRSs could be seen in both carriers and non-carriers of the APOE $\varepsilon 4$ allele. In stratified analyses, the $A P O E$ PRSs were associated with plasma p-tau181 in all diagnostic groups $(\mathrm{CU}, \mathrm{MCI}$, and $\mathrm{AD}$ dementia), while the non- $A P O E$ PRSs were associated with plasma p-tau181 only in the MCI group. The $A P O E$ PRSs showed similar results in $A \beta$ - positive and $\mathrm{A} \beta$-negative individuals, while the non- $A P O E$ PRSs were associated with plasma $p$-tau181 in $A \beta$ positives only. All results were similar when using a pre-calculated PHS available in the ADNI database.

So far, studies of the relation between AD-PRSs and plasma p-tau181 are lacking. Previous CSF-based results of most relevance for comparison with our plasma-based results are those presented in a recent study of polygenic burden on AD pathology in ADNI [16]. Similar to our finding, the authors reported that CSF p-tau181 was independently (after correction for $A P O E$ ) associated with the 31-SNP polygenic hazard score (PHS) (including $A P O E$ effects) used in our study. Neither results based on stratification by diagnostic status, nor by $A \beta$ pathology status, were presented.

In contrast to our findings, results in a paper on the longitudinal Australian Imaging, Biomarkers and Lifestyle (AIBL) Study of Ageing showed no association between a 21-SNP PRS and CSF p-tau levels in a small $(n=58)$ combined sample of cognitively normal and $\mathrm{AD}$ individuals [30]. This was the case both with and without inclusion of $A P O E$ in the PRS. When only considering cognitively normal individuals $(n=42)$, an association was seen with the non-APOE PRS. Another study reported no associations between a 19-SNP PRS (both with and without inclusion of $A P O E$ ) and CSF ptau levels in a sample of cognitively normal individuals enriched for a parental history of AD [31].

Other studies in relation to CSF are performed within $\mathrm{MCI}$ or AD only. A study on individuals with $\mathrm{MCI}$ showed a similar result as ours (i.e., an association with p-tau) when analyzing an 18-SNP non-APOE PRS in 391 individuals from two European cohorts [32]. In AD patients $(n=338)$, a study by Sleegers et al. [33] reported a correlation between a non-APOE 21-SNP PRS and CSF p-tau levels, while no association was seen when $A P O E$ was included in the score. In contrast, another study on AD patients $(n=890)$ [34], performing analyses with a relatively similar PRSs (including and excluding APOE), reported no associations with CSF p-tau. Both studies in $\mathrm{AD}$ contradict our results in the AD sub-group in ADNI (i.e., association with the $A P O E$ PRS and no association with the non- $A P O E$ PRS). In summary, studies of the relation between AD-PRSs and p-tau181 levels, including our novel plasma data, show mixed results, especially in cognitively normal and AD individuals. Possible explanations are differences in diagnostic/disease stage, and sometimes also differences in genetic variants included in the PRSs. Importantly, our results in plasma replicate a previous report in CSF within the same cohort [16]. Moreover, we included three versions of PRSs and they performed relatively similar. We also included the PHS, which was the polygenic score that performed best in relation to CSF biomarkers in the ADNI-study by Altmann et al. [16].

Our findings suggest that genetic risk for $\mathrm{AD}$, driven by $A P O E$, is associated with plasma p-tau181 in all diagnostic groups, while genetic risk for $\mathrm{AD}$ beyond $A P O E$ is associated with plasma p-tau181 only in the MCI group. Results from Karikari et al. [3] indicate that plasma ptau181 increases during early stages of tau pathology accumulation, but reaches a plateau in cases with moderate to severe pathology. In agreement, the performance of plasma p-tau to identify $\mathrm{AD}$ pathology is very high in later disease stages [7]. In view of these results, one could hypothesize that genetic risk factors for AD, especially those with smaller effect than $A P O E$, are of greater importance in relation to p-tau181 during the time when the level of the biomarker changes the most.

Considering the influence of $A \beta$ pathology on the relation between AD-PRS and plasma p-tau181, the PRSs (and PHS) including APOE seem to be associated with plasma p-tau181 independent of $A \beta$ pathology status, while the non- $A P O E$ scores were associated only in $A \beta$ positive individuals. One possible explanation may be that the relatively weak effect of the non- $A P O E$ PRSs in $\mathrm{AD}$ makes it easier to identify an association between the non-APOE PRSs and plasma $\mathrm{p}$-tau181 in $\mathrm{A} \beta$ positives, which constitute a genuine $\mathrm{AD}$ group harboring brain amyloidosis. In contrast, the very strong effect of $A P O E$ in $\mathrm{AD}$ may allow for identifying an association between the APOE PRSs and plasma p-tau181 levels also in a mixed population of cases with and without brain amyloidosis. Stratifying $\mathrm{A} \beta$-positive and $\mathrm{A} \beta$-negative 
individuals into diagnostic groups showed that the associations between the APOE PRSs and plasma p-tau181 were relatively independent of diagnostic status. The associations between the non-APOE PRSs and plasma ptau181 within $A \beta$ positives could, however, only be seen (at borderline significance level) in individuals with MCI. This indicates that having $\mathrm{MCI}$ and being $\mathrm{A} \beta$-positive is the most vulnerable combination in relation to the association between genetic risk for AD beyond $A P O E$ and plasma p-tau181. Previous studies have shown that ptau181 increases already in response to $A \beta$ pathology and then further in a stepwise manner with more advanced Braak stage $[3-5,7]$. P-tau181 signaling already in response to $A \beta$ positivity is in line with our results for the non- $A P O E$ PRSs (i.e., association with ptau181 in $\mathrm{MCI}$ and $\mathrm{A} \beta$ positive individuals).

Overall, our results support the importance of using biomarkers to identify $A \beta$ and tau pathology in studies assessing genetic risk for $\mathrm{AD}$. This study shows that we can learn more about the pathophysiology and disease processes in $\mathrm{AD}$ by combining information from wellvalidated fluid biomarkers and PRSs. High-risk individuals on the basis of genetics who do not increase in ptau as expected may be protected in some way; finding such resilience factors is important (and vice versa: low risk individuals who get $\mathrm{p}$-tau increase could have lifestyle-related risk factors). This will be important future studies, likely requiring larger cohorts. Further, utilizing information based on the combination of PRSs and blood biomarkers may be a useful way to detect and enrich clinical trials with individuals at higher risk to develop symptomatic AD.

\section{Limitations}

This study has some limitations that have to be addressed. The sample size is relatively small, making it difficult to find associations that survive correction for multiple testing in some of the stratified analyses. The majority of the associations seen in this study would remain after Bonferroni correction, but the associations with the non-APOE scores in $\mathrm{A} \beta$ positives would not and should therefore be interpreted with caution. Due to the lack of previous studies (i.e., previous effect sizes) of the relation between AD-PRSs and plasma p-tau181, a priori power analyses were not possible to do. However, post hoc power analyses based on effect sizes for the associations in our study showed that we had a relatively high power to detect these effect levels (using a $p$ value limit of $<0.05$ ), both in the total sample and after stratifying on either diagnostic or $A \beta$ pathology status. Still, stratifying on both diagnostic status and $A \beta$ pathology status generated small groups and these results have to be interpreted with caution. Further, the sample size of the different diagnostic groups differs. However, inspection of the $\beta$-values and standard errors indicates that associations seen in only one of the diagnostic groups (i.e., associations between the non-APOE PRSs, the PHS, and $\mathrm{MCI}$ ) are not merely a result of a larger sample size. Moreover, due to the very limited number of cohorts with both GWAS and plasma p-tau181 data, no replication sample of comparable size could be feasibly included in this study. Finally, the ADNI cohort could not be considered a representative populationbased sample, which limits the possibility of generalizing the results to the broader population.

\section{Conclusions}

Polygenic risk for AD including $A P O E$ was found to associate with plasma p-tau181 independent of diagnostic status and $A \beta$ pathology status, while polygenic risk for $\mathrm{AD}$ beyond $A P O E$ was associated with plasma p-tau181 only in MCI patients and $A \beta$-positive individuals. These results extend the knowledge about the relation between genetic risk for $\mathrm{AD}$ and $\mathrm{p}$-tau181 and further support the usefulness of plasma p-tau181 as a biomarker of AD.

\section{Supplementary Information}

The online version contains supplementary material available at https://doi. org/10.1186/s13195-020-00754-8.

\section{Additional file 1.}

\section{Abbreviations}

AD: Alzheimer's disease; ADNI: Alzheimer's Disease Neuroimaging Initiative; Aß: Amyloid- $\beta$; CSF: Cerebrospinal fluid; CU: Cognitively unimpaired; GWAS: Genome-wide association study; IBD: Identity-by-decent; LD: Linkage disequilibrium; MCl: Mild cognitive impairment; MAF: Minor allele frequency; p-tau181: Phosphorylated tau181; PHS: Polygenic hazard score;

PRS: Polygenic risk score; PET: Positron emission tomography; PCs: Principal components; Simoa: Single molecule array; SNPSs: Single nucleotide polymorphisms; SUVR: Standardized uptake value ratios

\section{Acknowledgements}

We thank the International Genomics of Alzheimer's Project (IGAP) for providing summary results data for these analyses. The investigators within IGAP contributed to the design and implementation of IGAP and/or provided data but did not participate in analysis or writing of this report. IGAP was made possible by the generous participation of the control subjects, the patients, and their families. The i-Select chips were funded by the French National Foundation on Alzheimer's disease and related disorders. EADI was supported by the LABEX (laboratory of excellence program investment for the future) DISTALZ grant, Inserm, Institut Pasteur de Lille, Université de Lille 2 and the Lille University Hospital. GERAD/PERADES was supported by the Medical Research Council (grant no. 503480), Alzheimer's Research UK (grant no. 503176), the Wellcome Trust (grant no. 082604/2/07/Z) and German Federal Ministry of Education and Research (BMBF): Competence Network Dementia (CND) grant no. 01G10102, 01GI0711, 01GI0420. CHARGE was partly supported by the NIH/NIA grant R01 AG033193 and the NIA AG081220 and AGES contract N01-AG-12100, the NHLBI grant R01 HL105756, the Icelandic Heart Association, and the Erasmus Medical Center and Erasmus University. ADGC was supported by the NIH/ NIA grants: U01 AG032984, U24 AG021886, U01 AG016976, and the Alzheimer's Association grant ADGC-10-196728.

\section{Authors' contributions}

Study design: AZ, JL, PP, HZ, and KB. Preparation of genetic data: JL and AZ. Biomarker analyses: NJA, ALB, TKK, JLR, AS, and MS-C. Statistical analyses: AZ. 
Drafting the manuscript: $A Z$ and $J$. Scientific input and reading the final version of the manuscript: All authors. All authors read and approved the final manuscript.

\section{Authors' information}

Not applicable.

\section{Funding}

KB is supported by the Swedish Research Council (\#2017-00915), the Alzheimer Drug Discovery Foundation (ADDF), USA (\#RDAPB-2018092016615), the Swedish Alzheimer Foundation (\#AF-742881), the Swedish Brain Foundation (\#FO2017-0243), the Swedish state Support for Clinical Research (\#ALFGBG-715986), and European Union Joint Program for Neurodegenerative Disorders (JPND2019-466-236). HZ is a Wallenberg Scholar supported by grants from the Swedish Research Council (\#201802532), the European Research Council (\#681712), Swedish State Support for Clinical Research (\#ALFGBG-720931), the Alzheimer Drug Discovery Foundation (ADDF), USA (\#201809-2016862), and the UK Dementia Research Institute at UCL. TKK holds a research fellowship from the BrightFocus Foundation (\#A2020812F) and is further supported by the Swedish Alzheimer Foundation (\#AF-930627), the Swedish Brain Foundation (\#FO2020-0240), the Swedish Dementia Foundation, Gamla Tjänarinnor Foundation, the Aina (Ann) Wallströms and Mary-Ann Sjöbloms Foundation, the Agneta PrytzFolkes \& Gösta Folkes Foundation (\#2020-00124), the Gun and Bertil Stohnes Foundation, and the Anna Lisa and Brother Björnsson's Foundation. MS-C received funding from the European Union's Horizon 2020 Research and Innovation Program under the Marie Sklodowska-Curie action grant agreement no. 752310 and currently receives funding from Instituto de Salud Carlos III (PI19/00155) and from the Spanish Ministry of Science, Innovation and Universities (Juan de la Cierva Programme grant IJC2018-037478-I). AS was supported by the Paulo Foundation and the Orion Research Foundation. AZ was supported by the Swedish Alzheimer foundation (\#AF-930582), The Swedish Dementia Foundation, Magnus Bergvalls Foundation, Gamla Tjänarinnor Foundation, and Hjalmar Svenssons Research Foundation. PP is an Alzheimer's Research UK Senior Research Fellow. JL is funded by the van Geest Endowment Fund. This paper represents independent research part-funded by the National Institute for Health Research (NIHR) Biomedical Research Centre at South London and Maudsley NHS Foundation Trust and King's College London. The views expressed are those of the authors and not necessarily those of the NHS, the NIHR, or the Department of Health and Social Care. Open Access funding provided by Gothenburg University Library.

\section{Availability of data and materials}

The data used in this article can be found in the ADNI database (http://adni. loni.usc.edu) [17].

\section{Ethics approval and consent to participate}

Regional ethical committees of all institutions included in ADNI approved of the study and all subjects have provided informed consent.

\section{Consent for publication}

Not applicable.

\section{Competing interests}

KB has served as a consultant, at advisory boards, or at data monitoring committees for Abcam, Axon, Biogen, JOMDD/Shimadzu. Julius Clinical, Lilly, MagQu, Novartis, Roche Diagnostics, and Siemens Healthineers, and is a cofounder of Brain Biomarker Solutions in Gothenburg AB (BBS), which is a part of the GU Ventures Incubator Program. $\mathrm{HZ}$ has served at scientific advisory boards for Denali, Roche Diagnostics, Wave, Samumed, Siemens Healthineers, Pinteon Therapeutics, and CogRx; has given lectures in symposia sponsored by Fujirebio, Alzecure, and Biogen; and is a co-founder of Brain Biomarker Solutions in Gothenburg AB (BBS), which is a part of the GU Ventures Incubator Program.

\section{Author details}

${ }^{1}$ Neuropsychiatric Epidemiology Unit, Department of Psychiatry and Neurochemistry, Institute of Neuroscience and Physiology, the Sahlgrenska Academy, Centre for Ageing and Health (AGECAP) at the University of Gothenburg, Gothenburg, Sweden. ${ }^{2}$ Maurice Wohl Clinical Neuroscience Institute, Institute of Psychiatry, Psychology and Neuroscience, King's College
London, London, UK. ${ }^{3}$ Department of Psychiatry and Neurochemistry, Institute of Neuroscience \& Physiology, the Sahlgrenska Academy at the University of Gothenburg, Mölndal, Sweden. ${ }^{4}$ Wallenberg Centre for Molecular and Translational Medicine, University of Gothenburg, Gothenburg, Sweden. ${ }^{5}$ Department of Old Age Psychiatry, Maurice Wohl Clinical Neuroscience Institute, King's College London, London, UK. ${ }^{6} \mathrm{NIHR}$ Biomedical Research Centre for Mental Health \& Biomedical Research Unit for Dementia at South London \& Maudsley NHS Foundation, London, UK. ${ }^{7}$ Translational Neuroimaging Laboratory, McGill Centre for Studies in Aging, McGill University, Montreal, QC, Canada. ${ }^{8}$ Turku PET Centre, University of Turku, Turku, Finland. ${ }^{9}$ Barcelonaßeta Brain Research Center (BBRC), Pasqual Maragall Foundation, Barcelona, Spain. ${ }^{10}$ IMIM (Hospital del Mar Medical Research Institute), Barcelona, Spain. ${ }^{11}$ Servei de Neurologia, Hospital del Mar, Barcelona, Spain. ${ }^{12}$ Centro de Investigación Biomédica en Red de Fragilidad y Envejecimiento Saludable (CIBERFES), Madrid, Spain. ${ }^{13} \mathrm{Clinical}$ Neurochemistry Laboratory, Sahlgrenska University Hospital, Mölndal, Sweden. ${ }^{14}$ Department of Neurodegenerative Disease, UCL Institute of Neurology, Queen Square, London, UK. ${ }^{15}$ UK Dementia Research Institute at UCL, London, UK.

\section{Received: 28 August 2020 Accepted: 16 December 2020}

\section{Published online: 08 January 2021}

\section{References}

1. Blennow K, de Leon MJ, Zetterberg H. Alzheimer's disease. Lancet. 2006; 368(9533):387-403.

2. Jack CR Jr, Bennett DA, Blennow K, Carrillo MC, Dunn B, Haeberlein SB, et al. NIA-AA Research Framework: toward a biological definition of Alzheimer's disease. Alzheimers Dement. 2018;14(4):535-62.

3. Karikari TK, Pascoal TA, Ashton NJ, Janelidze S, Benedet AL, Rodriguez JL, et al. Blood phosphorylated tau 181 as a biomarker for Alzheimer's disease: a diagnostic performance and prediction modelling study using data from four prospective cohorts. Lancet Neurol. 2020;19(5):422-33.

4. Janelidze S, Mattsson N, Palmqvist S, Smith R, Beach TG, Serrano GE, et al. Plasma P-tau181 in Alzheimer's disease: relationship to other biomarkers, differential diagnosis, neuropathology and longitudinal progression to Alzheimer's dementia. Nat Med. 2020;26(3):379-86.

5. Thijssen EH, La Joie R, Wolf A, Strom A, Wang P, laccarino L, et al. Diagnostic value of plasma phosphorylated tau181 in Alzheimer's disease and frontotemporal lobar degeneration. Nat Med. 2020;26(3):387-97.

6. O'Connor A, Karikari TK, Poole T, Ashton NJ, Lantero Rodriguez J, Khatun A, et al. Plasma phospho-tau181 in presymptomatic and symptomatic familial Alzheimer's disease: a longitudinal cohort study. Mol Psychiatry. 2020. Online ahead of print.

7. Lantero Rodriguez J, Karikari TK, Suarez-Calvet M, Troakes C, King A, Emersic A, et al. Plasma p-tau181 accurately predicts Alzheimer's disease pathology at least 8 years prior to post-mortem and improves the clinical characterisation of cognitive decline. Acta Neuropathol. 2020;140(3):267-78.

8. Kunkle BW, Grenier-Boley B, Sims R, Bis JC, Damotte V, Naj AC, et al. Genetic meta-analysis of diagnosed Alzheimer's disease identifies new risk loci and implicates A beta, tau, immunity and lipid processing (vol 51, pg 414, 2019). Nat Genet. 2019:51(9):1423-4.

9. Lambert JC, Ibrahim-Verbaas CA, Harold D, Naj AC, Sims R, Bellenguez C, et al. Meta-analysis of 74,046 individuals identifies 11 new susceptibility loci for Alzheimer's disease. Nat Genet. 2013;45(12):1452-8.

10. Jansen IE, Savage JE, Watanabe K, Bryois J, Williams DM, Steinberg S, et al. Genome-wide meta-analysis identifies new loci and functional pathways influencing Alzheimer's disease risk. Nat Genet. 2019;51(3):404-13.

11. Escott-Price V, Sims R, Bannister C, Harold D, Vronskaya M, Majounie E, et al. Common polygenic variation enhances risk prediction for Alzheimer's disease. Brain. 2015;138(Pt 12):3673-84.

12. Escott-Price $V$, Myers AJ, Huentelman M, Hardy J. Polygenic risk score analysis of pathologically confirmed Alzheimer disease. Ann Neurol. 2017; 82(2):311-4

13. Mormino EC, Sperling RA, Holmes AJ, Buckner RL, De Jager PL, Smoller JW, et al. Polygenic risk of Alzheimer disease is associated with early- and latelife processes. Neurology. 2016:87(5):481-8.

14. Scelsi MA, Khan RR, Lorenzi M, Christopher L, Greicius MD, Schott JM, et al. Genetic study of multimodal imaging Alzheimer's disease progression score implicates novel loci. Brain. 2018;141(7):2167-80. 
15. Sabuncu MR, Buckner RL, Smoller JW, Lee PH, Fischl B, Sperling RA, et al. The association between a polygenic Alzheimer score and cortical thickness in clinically normal subjects. Cereb Cortex. 2012;22(11):2653-61.

16. Altmann A, Scelsi MA, Shoai M, de Silva E, Aksman LM, Cash DM, et al. A comprehensive analysis of methods for assessing polygenic burden on Alzheimer's disease pathology and risk beyond APOE. Brain Commun. 2020; 2(1):fCZ047.

17. Alzheimer's Disease Neuroimaging Initiative (ADNI) database: http://adni. loni.usc.edu. Accessed May 1 to June 30, 2020.

18. Petersen RC, Aisen PS, Beckett LA, Donohue MC, Gamst AC, Harvey DJ, et al. Alzheimer's Disease Neuroimaging Initiative (ADNI): clinical characterization. Neurology. 2010;74(3):201-9.

19. Mattsson, N., U. Andreasson, H. Zetterberg, K. Blennow, and Alzheimer's Disease Neuroimaging Initiative, Association of plasma neurofilament light with neurodegeneration in patients with Alzheimer disease. JAMA Neurol 2017; 74(5): 557-566.

20. Karikari, T.K., A.L. Benedet, N.J. Ashton, J. Lantero Rodriquez, A. Snellman, M. Suárez-Calvet, et al. Diagnostic performance and prediction of clinical progression of plasma phospho-tau181 in the Alzheimer's Disease Neuroimaging Initiative. Mol Psychiatry. 2020. Online ahead of print.

21. Benedet AL, Ashton NJ, Pascoal TA, Leuzy A, Mathotaarachchi S, Kang MS, et al. Plasma neurofilament light associates with Alzheimer's disease metabolic decline in amyloid-positive individuals. Alzheimers Dement (Amst). 2019;11:679-89.

22. Saykin AJ, Shen L, Foroud TM, Potkin SG, Swaminathan S, Kim S, et al. Alzheimer's Disease Neuroimaging Initiative biomarkers as quantitative phenotypes: genetics core aims, progress, and plans. Alzheimers Dement. 2010;6(3):265-73.

23. Chang CC, Chow CC, Tellier LC, Vattikuti S, Purcell SM, Lee JJ. Secondgeneration PLINK: rising to the challenge of larger and richer datasets. Gigascience. 2015;4:7.

24. Price AL, Patterson NJ, Plenge RM, Weinblatt ME, Shadick NA, Reich D. Principal components analysis corrects for stratification in genome-wide association studies. Nat Genet. 2006;38(8):904-9.

25. The 1000 genomes project: https://www.internationalgenome.org/. Accessed 12020.

26. The Sanger Imputation Service: https://imputation.sanger.ac.uk/. Accessed 1 May 2020.

27. McCarthy S, Das S, Kretzschmar W, Delaneau O, Wood AR, Teumer A, et al. A reference panel of 64,976 haplotypes for genotype imputation. Nat Genet. 2016;48(10):1279-83.

28. de Rojas, I., S. Moreno-Grau, N. Tesi, B. Grenier-Boley, V. Andrade, I. Jansen, et al., Common variants in Alzheimer's disease: novel association of six genetic variants with $A D$ and risk stratification by polygenic risk scores. medRxiv. 2020: 19012021.

29. Desikan RS, Fan CC, Wang Y, Schork AJ, Cabral HJ, Cupples LA, et al. Genetic assessment of age-associated Alzheimer disease risk: development and validation of a polygenic hazard score. Plos Med. 2017;14(3):e1002258.

30. Porter T, Burnham SC, Milicic L, Savage G, Maruff P, Lim YY, et al. Utility of an Alzheimer's disease risk-weighted polygenic risk score for predicting rates of cognitive decline in preclinical Alzheimer's disease: a prospective longitudinal study. J Alzheimers Dis. 2018;66(3):1193-211.

31. Darst BF, Koscik RL, Racine AM, Oh JM, Krause RA, Carlsson CM, et al. Pathway-specific polygenic risk scores as predictors of amyloid-beta deposition and cognitive function in a sample at increased risk for Alzheimer's disease. J Alzheimers Dis. 2017;55(2):473-84.

32. Louwersheimer E, Wolfsgruber S, Espinosa A, Lacour A, Heilmann-Heimbach S, Alegret $\mathrm{M}$, et al. Alzheimer's disease risk variants modulate endophenotypes in mild cognitive impairment. Alzheimers Dement. 2016;12(8):872-81.

33. Sleegers K, Bettens K, De Roeck A, Van Cauwenberghe C, Cuyvers E, Verheijen J, et al. A 22-single nucleotide polymorphism Alzheimer's disease risk score correlates with family history, onset age, and cerebrospinal fluid Abeta42. Alzheimers Dement. 2015;11(12):1452-60.

34. Martiskainen H, Helisalmi S, Viswanathan J, Kurki M, Hall A, Herukka SK, et al. Effects of Alzheimer's disease-associated risk loci on cerebrospinal fluid biomarkers and disease progression: a polygenic risk score approach. J Alzheimers Dis. 2015;43(2):565-73.

\section{Publisher's Note}

Springer Nature remains neutral with regard to jurisdictional claims in published maps and institutional affiliations.

\section{Ready to submit your research? Choose BMC and benefit from}

- fast, convenient online submission

- thorough peer review by experienced researchers in your field

- rapid publication on acceptance

- support for research data, including large and complex data types

- gold Open Access which fosters wider collaboration and increased citations

- maximum visibility for your research: over $100 \mathrm{M}$ website views per year

At BMC, research is always in progress.

Learn more biomedcentral.com/submissions 